\title{
Transcriptomic Analysis of Dark-Induced Senescence in Bermudagrass (Cynodon dactylon)
}

\author{
Jibiao Fan ${ }^{1,2,3}$, Yanhong Lou ${ }^{4}$, Haiyan Shi ${ }^{5}$ (D), Liang Chen ${ }^{2,3, *}$ and Liwen Cao ${ }^{2,3, *(1)}$ \\ 1 College of Animal Science and Technology, Yangzhou University, Yangzhou 225009, China; \\ 006298@yzu.edu.cn \\ 2 CAS Key Laboratory of Plant Germplasm Enhancement and Specialty Agriculture, Wuhan Botanical Garden, \\ Chinese Academy of Sciences, Wuhan 430074, China \\ 3 Center of Economic Botany, Core Botanical Gardens, Chinese Academy of Sciences, Wuhan 430074, China \\ 4 National Engineering Laboratory for Efficient Utilization of Soil and Fertilizer Resources, College of \\ Resources and Environment, Shandong Agricultural University, Daizong Road, Tai'an 271018, China; \\ yanhonglou@sdau.edu.cn \\ 5 College of Horticulture, Agricultural University of Hebei, Baoding 071001, China; yyshhy@hebau.edu.cn \\ * Correspondence: chenliang888@wbgcas.cn (L.C.); caoliwen@wbgcas.cn (L.W.C.)
}

Received: 9 November 2019; Accepted: 12 December 2019; Published: 17 December 2019

\begin{abstract}
Leaf senescence induced by prolonged light deficiency is inevitable whenever turfgrass is cultivated in forests, and this negatively influences the survival and aesthetic quality of the turfgrass. However, the mechanism underlying dark-induced senescence in turfgrass remained obscure. In this study, RNA sequencing was performed to analyze how genes were regulated in response to dark-induced leaf senescence in bermudagrass. A total of 159,207 unigenes were obtained with a mean length of $948 \mathrm{bp}$. The differential expression analysis showed that a total of 59,062 genes, including 52,382 up-regulated genes and 6680 down-regulated genes were found to be differentially expressed between control leaves and senescent leaves induced by darkness. Subsequent bioinformatics analysis showed that these differentially expressed genes (DEGs) were mainly related to plant hormone (ethylene, abscisic acid, jasmonic acid, auxin, cytokinin, gibberellin, and brassinosteroid) signal transduction, N-glycan biosynthesis, and protein processing in the endoplasmic reticulum. In addition, transcription factors, such as WRKY, NAC, HSF, and bHLH families were also responsive to dark-induced leaf senescence in bermudagrass. Finally, qRT-PCR analysis of six randomly selected DEGs validated the accuracy of sequencing results. Taken together, our results provide basic information of how genes respond to darkness, and contribute to the understanding of comprehensive mechanisms of dark-induced leaf senescence in turfgrass.
\end{abstract}

Keywords: transcriptome; senescence; darkness; bermudagrass

\section{Introduction}

Cultivating turfgrass in forests has become a necessity of developing forestry economy and constructing gardens and landscapes. However, light deprivation caused by the forests exerts many negative effects on the turfgrass. When turfgrass is exposed to light-deficiency conditions, a series of morphological changes happen, including an increase in shoot elongation, leaf length, and leaf area [1-3]. Leaf senescence will be induced by prolonged and severe light deficiency. Leaf senescence is a critical concern for turfgrass because it negatively influences not only plant growth and development, but also the aesthetic turf quality. Therefore, it is important to investigate the mechanisms underlying dark-induced leaf senescence in order to develop ways to delay leaf senescence and improve turf quality under environments of light deficiency. 
Leaf senescence is a complex and natural process at the final stage of leaf development, involving a series of changes in cellular physiology and biochemistry, enzyme activity, and gene expression [4]. Leaf senescence can as well be induced by abiotic stresses, such as darkness [5]. Previous studies have indicated that there is some overlap between natural senescence and dark-induced senescence [6]. Most of the genes that depend on ethylene and jasmonic acid (JA) for expression during natural leaf senescence also showed up-regulation during dark-induced senescence, indicating that these two pathways have an active signaling role in both age and dark-induced leaf senescence in Arabidopsis [6]. In rice plants, among 14 senescence-associated gene (SAG) clones characterized, 11 were found to be associated with both dark-induced and natural leaf senescence [7]. Several key age-dependent SAGs have been demonstrated to regulate dark-induced leaf senescence as well, such as NON-YELLOW COLORING1/3 (NYC1/3), EARLY FLOWERING3.1 (ELF3.1), NAP, ORESARA1 (ORE1), and DNA-binding with one finger 2.1 (Dof2.1) [8-15].

Despite all this, dark-induced and age-dependent senescence still triggers different gene expression profiles and signaling pathways $[6,16,17]$. Recent studies have demonstrated that the phytochrome-interacting factors (PIFs) signaling module plays a master role in dark-induced leaf senescence [18]. In Arabidopsis, PIF4/PIF5 are central and necessary transcriptional activators of dark-induced leaf senescence. They are induced by darkness in a phytochrome B (phyB)-dependent manner. These two PIFs finally regulate chloroplast maintenance, hormone signaling, chlorophyll metabolism, and senescence master regulators during dark-induced senescence via multiple coherent feed-forward loops, together with ORE1, ETHYLENE INSENSITIVE 3 (EIN3), and ABA INSENSITIVE 5 (ABI5) and ENHANCED EM LEVEL (EEL) [19-21]. Light deprivation can result in a reduction in photosynthesis and subsequent carbon starvation. Some transcription factors involved in low-energy response also play important regulatory-roles in dark-induced leaf senescence. For example, overexpression of BASIC LEUCINE ZIPPER63 (bZIP63) or bZIP1 which were responsive to low energy could accelerate dark-induced leaf senescence [22,23].

Bermudagrass (Cynodon dactylon L.), frequently cultivated in sports fields, home lawns, and golf courses, is one of the most widely used warm-season turfgrass species [24]. However, dark-induced leaf senescence has not been studied yet in bermudagrass. Therefore, it is necessary to investigate the genome-wide response to dark stress, the most severe light-deficiency condition, in bermudagrass. RNA-sequencing is an important and direct approach to compare gene expression, and discover novel and rare transcripts in plants [25-27]. Identifying genes that are specifically responsive to dark-induced senescence by RNA-sequencing is the first and key step to explore the underlying mechanism of dark-induced senescence in bermudagrass. Therefore, in this study, prolonged dark treatment for the whole bermudagrass plants was used to initiate leaf senescence under laboratory conditions to mimic leaf senescence induced by light deficiency under natural conditions. Transcriptome analysis was then performed between senescent leaves induced by prolonging darkness and control leaves in bermudagrass. The objective of this study was to identify candidate genes and pathways involved in the dark-induced senescence process, and to provide basic information for elucidating the molecular mechanisms underlying dark-induced leaf senescence in bermudagrass.

\section{Results}

\subsection{Dark Induced Leaf Senescence in Bermudagrass}

To determine the effect of darkness on leaf senescence in bermudagrass, phenotypes of bermudagrass under dark treatment and control condition were examined. After $7 \mathrm{~d}$ of dark treatment (DT), leaf senescence initiated from the bottom, and severely yellowing leaves were observed in the bottom leaves (Figure 1a,b). However, all leaves of control plants (CK) still kept green. In addition, other senescence symptoms were also measured. Consistently, chlorophyll contents (Figure 1c) and PSII max-photo efficiencies (Fv/Fm) (Figure 1d) of the bottom leaves both declined after $7 \mathrm{~d}$ of dark treatment. However, there were no significant changes in bottom leaves in control plants after $7 \mathrm{~d}$. 
(a)

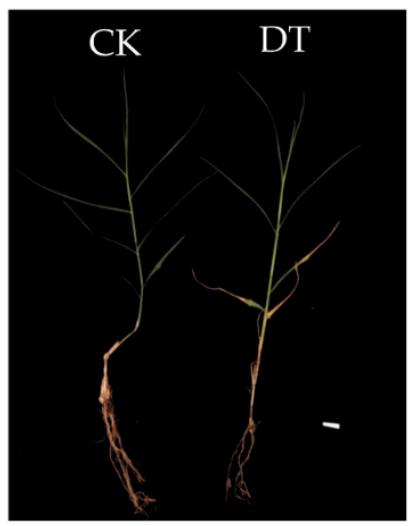

(c) (b)

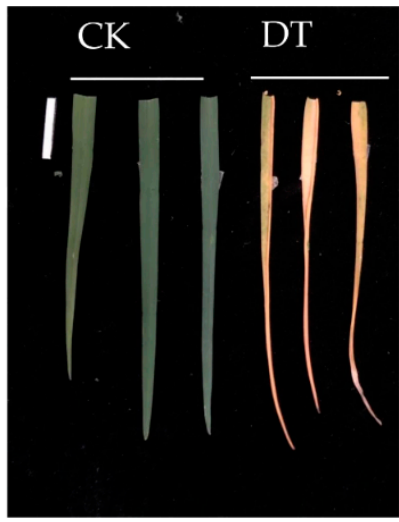

(d)

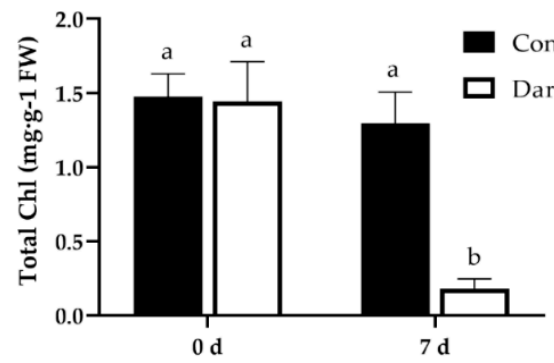

Days after dark treatments

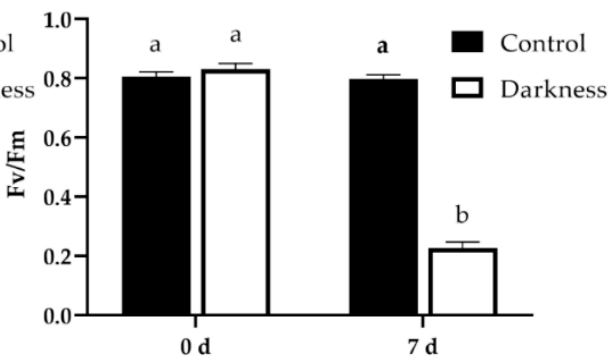

Days after dark treatments

Figure 1. Phenotype analysis of bermudagrass after dark treatment. (a) Phenotype of whole plants after $7 \mathrm{~d}$ of control (left: CK) and dark (right: DT) treatments (scale $=1 \mathrm{~cm}) ;(\mathbf{b})$ phenotype of bottom leaves after $7 \mathrm{~d}$ of control (left: CK) and dark (right: DT) treatments (scale $=1 \mathrm{~cm}) ;(\mathbf{c}, \mathbf{d})$ total chlorophyll contents (c) and Fv/Fm (d) of the first and second leaves from bermudagrass under control and dark treatments. Significant $(p<0.05)$ differences are indicated by different letters using Tukey's HSD test. Error bars indicate the SD of three biological repeats. Fw-fresh weight.

\subsection{Characteristics of Genes in Response to Dark-Induced Leaf Senescence in Bermudagrass}

To investigate genes responsive to dark-induced senescence in bermudagrass, a total of six cDNA libraries constructed from leaves with or without dark treatment $(7 \mathrm{~d})$ were sequenced using the Illumina Hiseq. After removing the low-quality reads and adaptors, 42,557,344; 53,558,496; 58,132,180; $64,587,208 ; 52,195,444$, and 60,166,630 clean reads (>99\%) were obtained for CK_1,CK_2,CK_3, DT_1, DT_2, and DT_3, respectively (Table 1). Subsequently, these high-quality clean reads were assembled using the Trinity de novo assembly program because of the absence of reference genome sequences. A total of 387,221 transcripts and 159,207 unigenes were obtained with a mean length of $1090 \mathrm{bp}$ and $948 \mathrm{bp}$, respectively (Table 2). The results showed that sequencing quality was sufficiently high for downstream analyses.

Table 1. Characteristics of RNA-sequencing results of all samples.

\begin{tabular}{cccccccc}
\hline Sample & Raw Reads & Clean Reads & Clean Bases & Error (\%) & Q20(\%) $^{\mathbf{1}}$ & Q30(\%) $^{\mathbf{2}}$ & GC(\%) $^{\mathbf{3}}$ \\
\hline CK_1 & $45,224,598$ & $42,557,344$ & 6.38 & 0.03 & 97.90 & 94.07 & 51.53 \\
CK_2 & $55,052,156$ & $53,558,496$ & 8.03 & 0.02 & 98.21 & 94.71 & 53.28 \\
CK_3 & $59,896,392$ & $58,132,180$ & 8.72 & 0.02 & 98.35 & 95.04 & 53.44 \\
DT_1 & $67,686,056$ & $64,587,208$ & 9.69 & 0.02 & 98.03 & 94.40 & 52.53 \\
DT_2 & $54,946,418$ & $52,195,444$ & 7.83 & 0.02 & 98.10 & 94.51 & 52.67 \\
DT_3 & $65,845,158$ & $60,166,630$ & 9.02 & 0.02 & 98.00 & 94.31 & 52.34 \\
\hline
\end{tabular}

${ }^{1} \mathrm{Q} 20(\%)$ is the proportion of nucleotides with a quality value larger than $20 .{ }^{2} \mathrm{Q} 30(\%)$ is the proportion of nucleotides with a quality value larger than $30 .{ }^{3} \mathrm{GC}(\%)$ is the proportion of guanidine and cytosine nucleotides among the total nucleotides. 
Table 2. The number of the transcripts and unigenes with different kinds of length clustered from the de novo assembly.

\begin{tabular}{|c|c|c|}
\hline Category & Transcripts & Unigenes \\
\hline $200-500 \mathrm{bp}$ & 128,446 & 60,507 \\
\hline $500-1 \mathrm{kbp}$ & 111,291 & 53,622 \\
\hline $1-2 \mathrm{kbp}$ & 97,001 & 30,181 \\
\hline$>2 \mathrm{kbp}$ & 50,483 & 14,897 \\
\hline Min length (bp) ${ }^{1}$ & 301 & 301 \\
\hline Mean length (bp) & 1090 & 948 \\
\hline Max length (bp) ${ }^{2}$ & 53,494 & 53,494 \\
\hline N50 length (bp) ${ }^{3}$ & 1559 & 1284 \\
\hline N90 length (bp) 4 & 465 & 426 \\
\hline Total & 387,221 & 159,207 \\
\hline
\end{tabular}

\footnotetext{
${ }^{1}$ The Min length means the minimum length of transcripts or unigenes. ${ }^{2}$ The Max length means the maximum length of transcripts and unigenes ${ }^{3}$ The N50 value is defined as the contig length where half of the assembly is represented by contigs of this size or longer. ${ }^{4}$ The N90 value is defined as the contig length where $90 \%$ of the assembly is represented by contigs of this size or longer.
}

\subsection{Gene Annotation of the Bermudagrass Transcriptome}

To analyze function of the 159,207 unigenes of bermudagrass, functional annotation of all unigenes was investigated using seven databases, including non-redundant protein sequence database $(\mathrm{Nr})$, nucleotide sequence database (Nt), Pfam, clusters of orthologous groups of proteins (KOG/COG), Swiss-Prot, Kyoto encyclopedia of genes and genomes (KEGG), and gene ontology (GO). In total, there were 119,581 unigenes $(75.11 \%)$ successfully annotated in at least one database, with 6961 unigenes $(4.37 \%)$ in all seven databases (Table 3$)$.

Table 3. The numbers and distribution rate of unigenes in the databases of the non-redundant protein sequence (Nr), nucleotide sequence (Nt), Kyoto encyclopedia of genes and genomes (KEGG), Swiss-Prot, Pfam, gene ontology (GO), and clusters of orthologous groups of proteins (KOG/COG).

\begin{tabular}{ccc}
\hline Categories & Number of Unigenes & Percentage (\%) \\
\hline Annotated in NR & 39,336 & 24.7 \\
Annotated in NT & 76,881 & 48.28 \\
Annotated in KEGG & 36,014 & 22.62 \\
Annotated in Swiss-Prot & 83,705 & 52.57 \\
Annotated in PFAM & 84,295 & 52.94 \\
Annotated in GO & 84,295 & 52.94 \\
Annotated in KOG & 41,079 & 25.8 \\
Annotated in all Databases & 6961 & 4.37 \\
Annotated in at least one Database & 119,581 & 75.11 \\
Total Unigenes & 159,207 & 100 \\
\hline
\end{tabular}

$\mathrm{Nr}$, non-redundant protein sequence database; $\mathrm{Nt}$, nucleotide sequence database; KEGG, Kyoto encyclopedia of genes and genomes; KOG, clusters of orthologous groups of proteins; GO, gene ontology.

For GO analysis, genes involved in 'metabolic process' $(46,108)$, 'cellular process' $(47,512)$, and 'single-organism process' $(38,511)$ were highly represented in the biological process. In terms of the cellular component category, 'cell' $(24,404)$, 'cell part' $(24,404)$, and 'organelle' $(15,635)$ were highly enriched. Within the molecular function category, the major sub-categories were 'binding' $(42,184)$, 'catalytic activity' $(39,993)$, and 'transporter activity' (6464).

For KOG analysis, a total of 41,079 enriched unigenes were divided into 25 groups. The largest group was 'posttranslational modification, protein turnover, chaperones' (5483), followed by 'general function prediction only' (5130), and then 'energy production and conversion' (3949).

For KEGG analysis, 36,014 unigenes were classified into five main biochemical pathways: 'cellular processes', 'environmental information processing', 'genetic information processing', 'metabolism', and 
'organismal systems'. Within the metabolism pathway, 'overview', 'carbohydrate metabolism', and 'amino acid metabolism' were prominently represented. The pathways related to 'genetic information processing' included 'folding, sorting, and degradation', 'replication and repair', 'transcription', and 'translation'. All function annotations provided important information for analyzing the processes, and pathways involved in dark-induced leaf senescence.

\subsection{Differentially Expressed Genes (DEGs) in Response to Dark-Induced Leaf Senescence in Bermudagrass}

To investigate the global gene expressions in response to dark-induced leaf senescence, the differentially expressed genes between the control and dark-treated samples were analyzed. According to the criteria that at least a two-fold difference and an adjusted $p$-value less than 0.05 $(|\log 2(\mathrm{DT} / \mathrm{CK})| \geq 1$, padj $\leq 0.05)$, a total of 59,062 genes, including 52,382 up-regulated genes and 6680 down-regulated genes, were found to be differentially expressed between CK and DT (Figure 2). It should be noted that the number of up-regulated genes was much larger than that of down-regulated genes, indicating that most of the genes were induced after dark treatment.

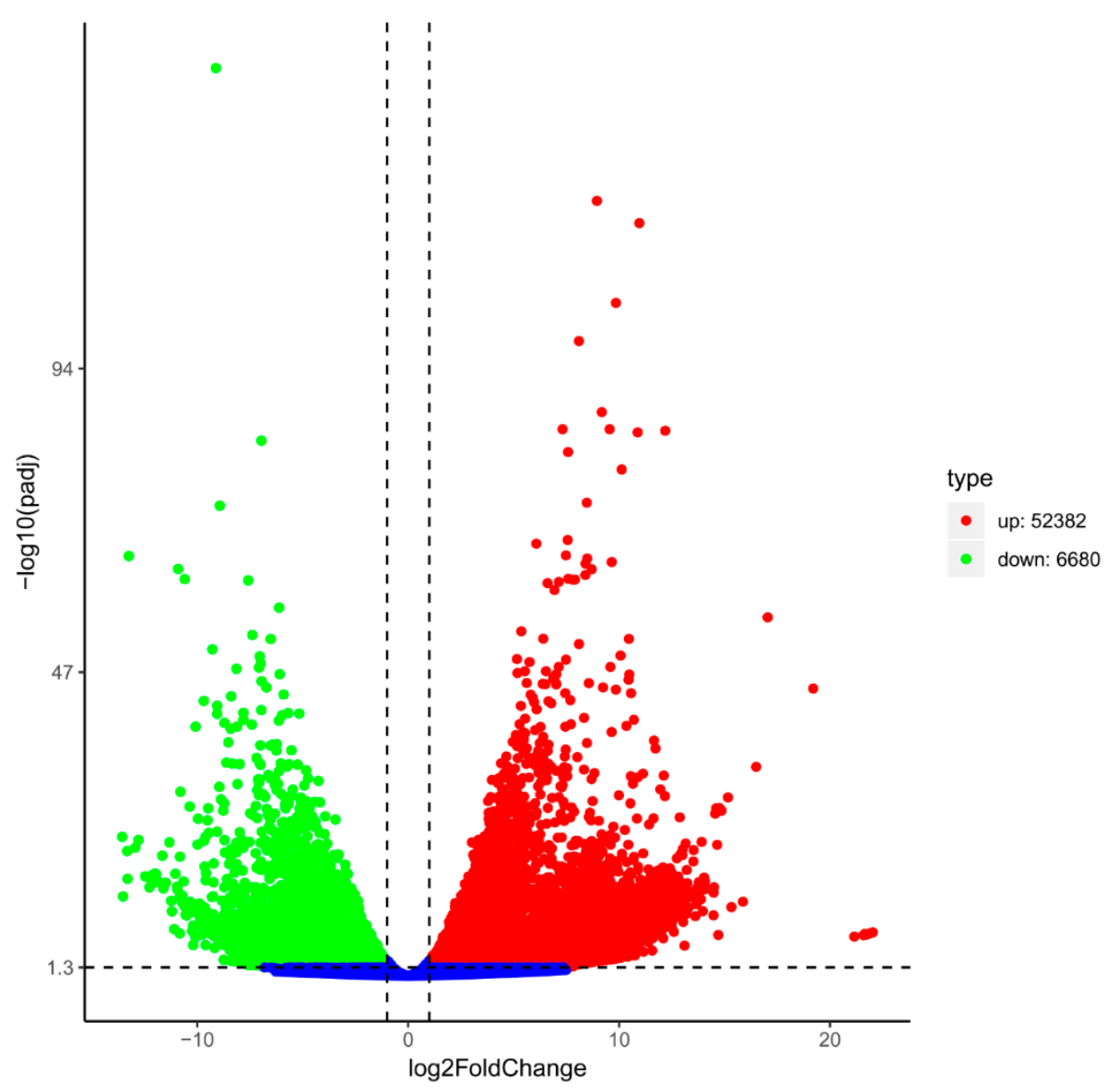

Figure 2. Volcano plot of transcriptional differences in Bermudagrass during dark-induced senescence. The vertical lines correspond to 2.0-fold up and down, and the horizontal line represents an adjusted $p$-value of 0.05 . Red dots represent up-regulated differentially expressed genes (DEGs), and green dots represent down-regulated DEGs. DEGs, differentially expressed genes.

\subsection{GO Analysis of Differentially Expressed Genes between CK and DT}

To determine the biological processes of dark-responsive DEGs, GO analysis of DEGs between CK and DT was performed. A total of 32,383 DEGs could be assigned as a GO term and were annotated into 20 biological process items, 20 cellular component items, and 20 molecular function items (Figure 3, Table S1). Within the biological process category, translation, peptide biosynthetic 
process, and peptide metabolic process were the most enriched groups. Within the cellular component category, unigenes were prominently enriched in the cytoplasm part, cytoplasm, and intracellular organelle. In the molecular function category, unigenes were classified into a structural constituent of ribosome, structural molecule activity, and threonine-type endopeptidase activity.

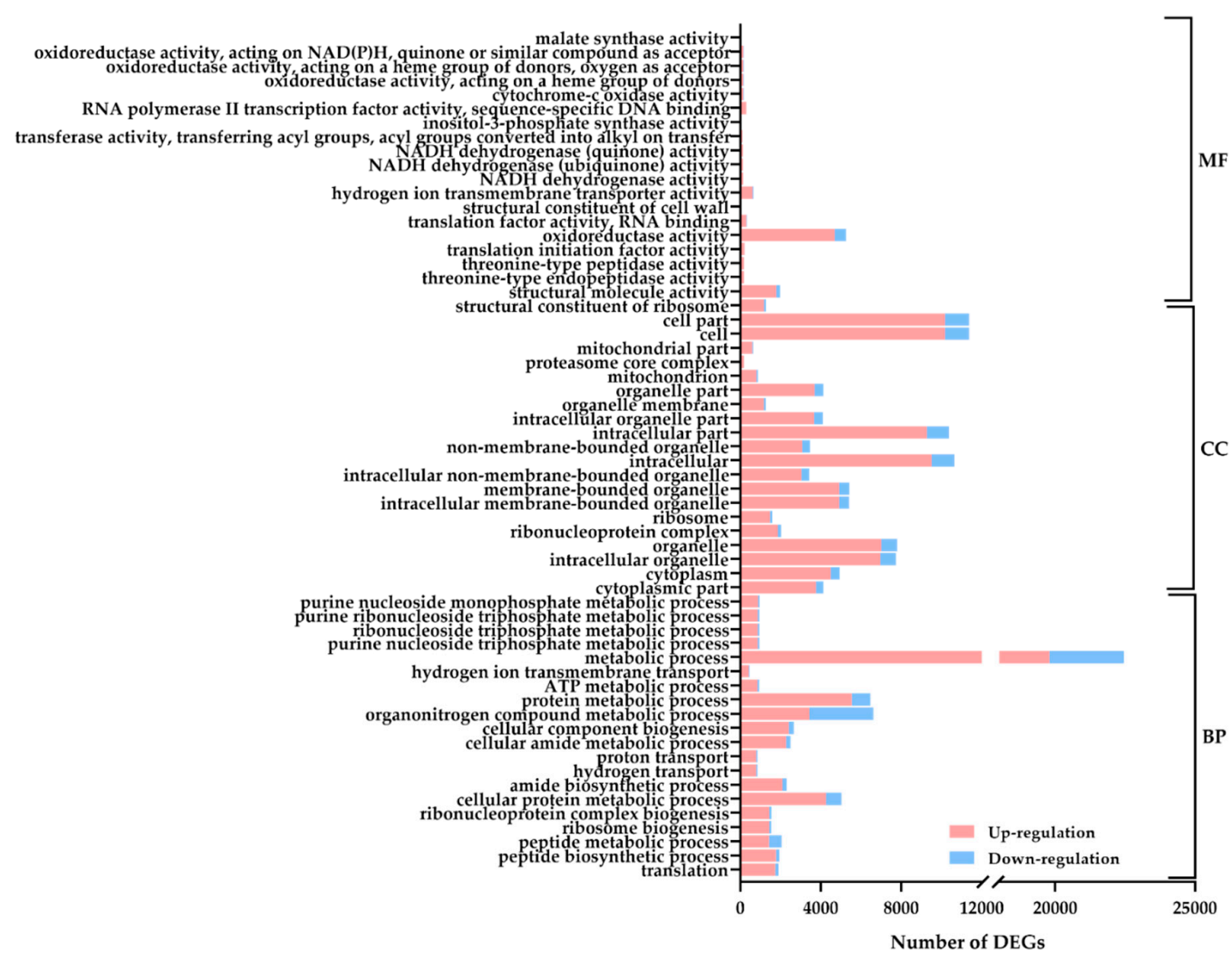

Figure 3. Gene Ontology (GO) classification of DEGs between CK and DT. DEGs were annotated in three categories: biological process (BP), cellular component (CC), and molecular function (MF). The enrichment index of each ontology term increased from the bottom up along Y-axis in all three categories.

\subsection{KEGG Pathway Enrichment Analysis of DEGs between CK and DT}

To further explore the biological functions of the DEGs between CK and DT, KEGG pathway enrichment analysis was conducted (Figure S1). The up-regulated DEGs were annotated into 122 KEGG pathways. The significantly enriched pathways were ribosome, citrate cycle (TCA cycle), proteasome, oxidative phosphorylation, phagosome, protein processing in the endoplasmic reticulum, regulation of autophagy, endocytosis, pentose phosphate pathway, glycolysis/gluconeogenesis, and spliceosome. However, the down-regulated DEGs were involved in completely different pathways, such as photosynthesis, photosynthesis-antenna proteins, phenylpropanoid biosynthesis, plant hormone signal transduction, carotenoid biosynthesis, carbon fixation in photosynthetic organisms, fatty acid elongation, cyanoamino acid metabolism, and porphyrin and chlorophyll metabolism.

\subsection{Genes Responsive to Dark-Induced Leaf Senescence in Bermudagrass}

Plant hormone signal transduction. Plant hormones have been reported to play important roles either in natural leaf senescence or in dark-induced leaf senescence. Here, genes involved in plant hormone signal transduction pathways were differentially expressed during dark-induced leaf senescence in bermudagrass (Figure 4). Ethylene is thought to be a senescence-promoting phytohormone. Key components of the ethylene signal transduction pathway were almost all 
up-regulated after dark treatment, including six ETHYLENE RESPONSES (ETRs), one CONSTITUTIVE TRIPLE RESPONSE 1 (CTR1), 17 MITOGEN-ACTIVATED PROTEIN KINASE 6 (MPK6), one EIN2, one EIN3-BINDING F-BOX PROTEIN 1/2 (EBF1/2), and one ETHYLENE RESPONSE FACTOR 1/2 (ERF1/2). Only one ERF was down-regulated. Abscisic acid (ABA) level increases with dark-induced leaf senescence. DEGs involved in ABA signal transduction were also highly represented and most unigenes were up-regulated as well. The expression of one PYL, 11 TYPE 2C PROTEIN PHOSPHATASES (PP2Cs), nine SUCROSE NONFERMENTING 1-RELATED PROTEIN KINASE 2S (SnRK2s), and two ABA-RESPONSIVE ELEMENT-BINDING FACTORS (ABFs) were up-regulated during dark-induced leaf senescence. For salicylic acid, there was one up-regulated PATHOGENESIS-RELATED GENES 1 (NPR1), ten up-regulated TGACG SEQUENCE-SPECIFIC BINDING PROTEINS (TGAs), ten up-regulated PATHOGENESIS-RELATED PROTEIN 1s (PR-1s), and one down-regulated NPR1 after dark treatment. JA is critically involved in senescence. Application of JA induces premature senescence and the level of JA increases with leaf senescing. During the dark-induced leaf senescence, several genes involved in JA signaling or response were differentially regulated. JASMONATE RESISTANT (JAR) and CORONATINE-INSENSITIVE PROTEIN 1 (COI1) genes were both down-regulated. As the direct target of COI1, all JASMONATE ZIM-DOMAIN (JAZ) genes were up-regulated as expected. Exogenous application or endogenous overexpression of cytokinin can delay leaf senescence. Although no genes involved in cytokinin biosynthesis were identified with differential expression, several genes involved in cytokinin signaling pathway (A multistep ARABIDOPSIS HISTIDINE KINASE (AHK) $\rightarrow$ ARABIDOPSIS HISTIDINE-CONTAINING PHOSPHOTRANSMITTER (AHP) $\rightarrow$ ARABIDOPSIS THALIANA RESPONSE REGULATORS (ARR) phosphorelay pathway) were up- or down-regulated during dark-induced senescence. Only one $A H K 4$ and one $A H P$ were detected with down-regulation. One A-type $A R R$ genes and two B-type $A R R$ genes were suppressed, whereas three A-type $A R R$ genes and one B-type $A R R$ genes were induced. The role of auxin in leaf senescence is still controversial. AUXIN RESPONSE FACTOR (ARF) and AUXIN/INDOLE-3-ACETIC ACID (Aux/IAA) proteins are crucial factors in the auxin response pathway. Expression of almost all $A R F$ genes was decreased in the senescent leaves induced by dark except for that of $A R F 16$. Similar to the $A R F s, 3 / 4$ of the $A u x / I A A$ genes are down-regulated during dark-induced senescence. In addition, genes participating in signal transduction of gibberellin, and brassinosteroid were also regulated during dark-induced senescence (Table S2).

Protein processing in the endoplasmic reticulum. Up-regulated DEGs were significantly enriched in protein processing in the endoplasmic reticulum pathway. Among genes involved in protein processing in the endoplasmic reticulum, one of the most conspicuous changes was the up-regulation of heat shock proteins (Hsps). Hsps were reported to play key roles in aging phenotypes in animals [28]. However, little is known about their roles in leaf senescence, especially dark-induced leaf senescence. During dark treatment, a total of 221 Hsps were up-regulated, including Hsp20s, Hsp70s, and Hsp90s (Figure 5a). Only nine Hsps were suppressed after $7 \mathrm{~d}$ of dark treatment.

$N$-glycan biosynthesis. It is worth noting that dark-induced leaf senescence activated almost all genes participating in N-glycan biosynthesis. A total of 109 DEGs were involved in N-glycan biosynthesis (Figure 5b), 107 of which were up-regulated, including ASPARAGINE-LINKED GLYCOSYLATION1,2,3,4,6,7,9,11,13,14 (ALG1,2,3,5,6,7,9,11,13,14), DOLICHOL-PHOSPHATE MANNOSYLTRANSFERASE 1,2,3 (DPM1,2,3), STAUROSPORIN AND TEMPERATURE SENSITIVE (STT), OLIGOSACCHARYLTRANSFERASE (OST), MANNOSYL-OLIGOSACCHARIDE GLUCOSIDASE 1 (GCS1), MANNOSYL-OLIGOSACCHARIDE ALPHA-1,2-MANNOSIDASE (MAN1), MANNOSYL-OLIGOSACCHARIDE ALPHA-1,3-GLUCOSIDASE (GANAB), and ALPHA-1,6-MANNOSYL-GLYCOPROTEIN BETA-1,2-N-ACETYLGLUCOSAMINYLTRANSFERASE (MGAT2). Only one OST and one MGAT3 were down-regulated during dark-induced senescence. 


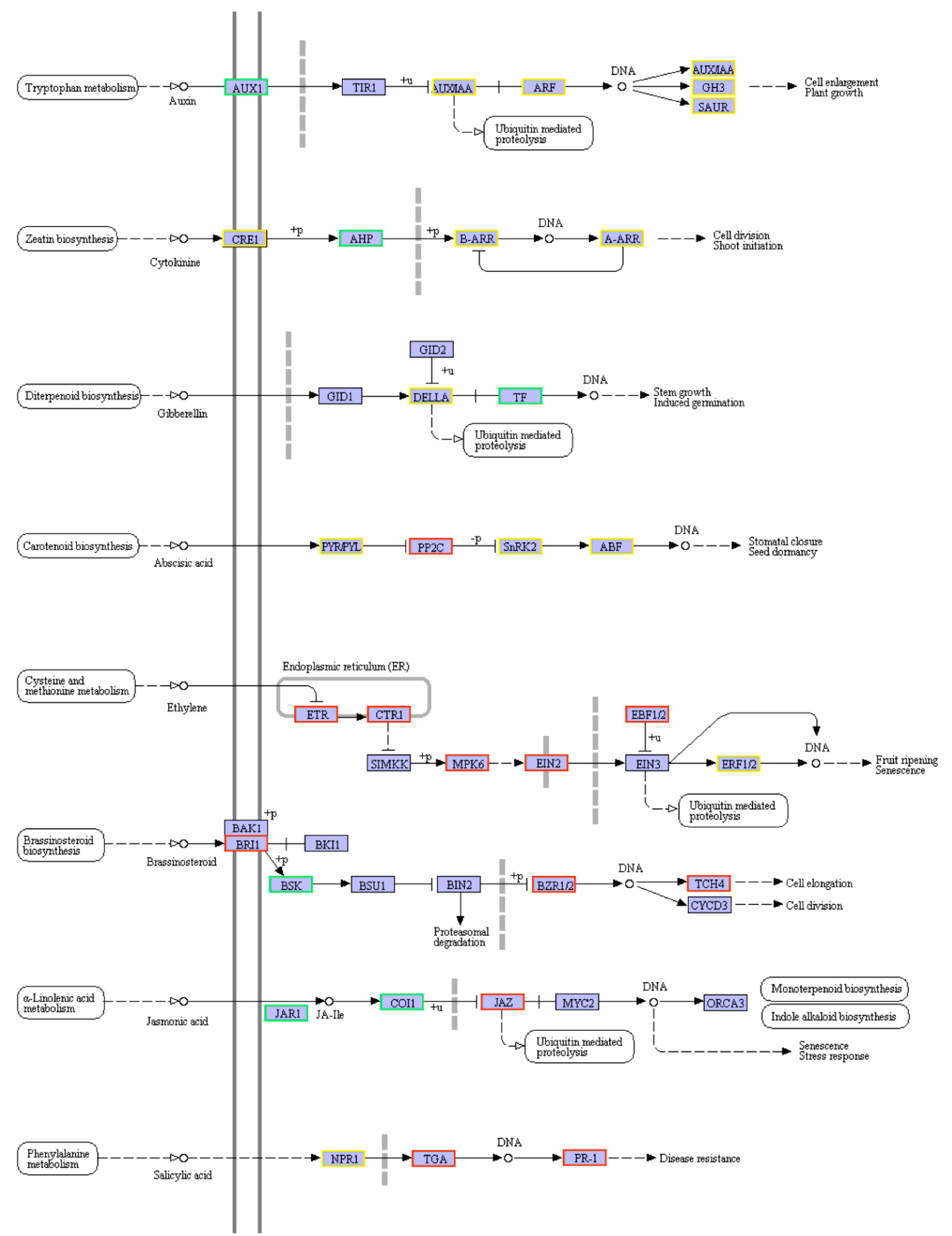

Figure 4. Differentially expressed genes between CK and DT in plant hormone signal transduction pathway. The red frame represents up-regulated genes, green frame represents down-regulated genes, and the yellow frame represents both up-regulated and down-regulated genes. 

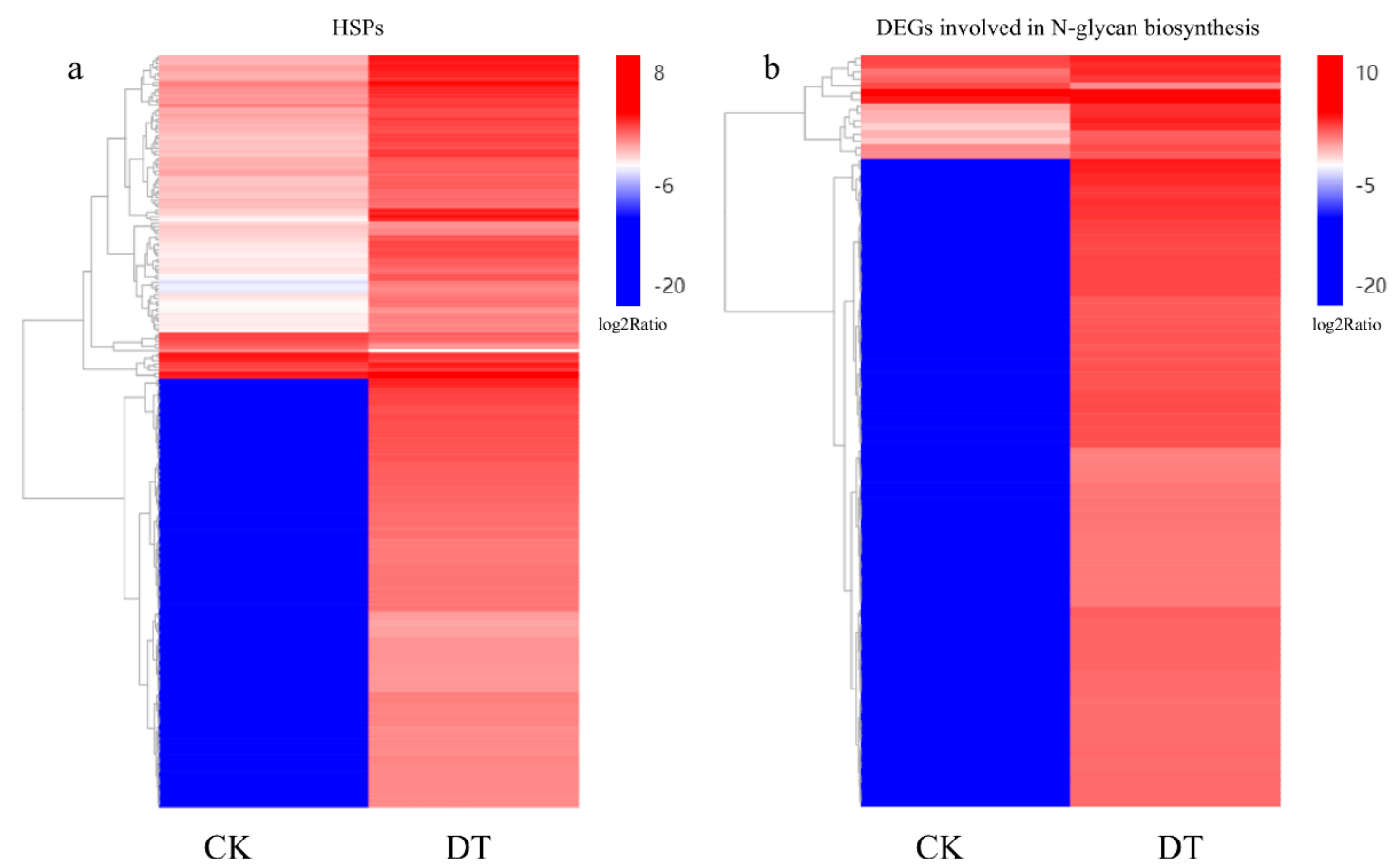

Figure 5. Hierarchical clustering analysis of DEGs between CK and DT. (a) Heat shock proteins (Hsps) were largely and significantly induced by dark-induced leaf senescence; (b) Almost all DEGs involved in N-glycan biosynthesis were activated during dark-induced leaf senescence. Different color bands represent the different expression level of each sample. The red bands represent a high expression level and the blue bands represent low expression levels.

\subsection{Regulation of Transcription Factor (TF) Families during Dark-Induced Leaf Senescence}

NAC and WRKY are two of the most important transcription factor families in the regulation of leaf senescence [8,9,29-33]. In this study, members of various NAC (Table S3) and WRKY TFs (Table S4) were identified during dark-induced senescence. Overall, the number of up-regulated WRKY and NAC TFs was greater than that of down-regulated ones. Of the DEGs encoding NAC and WRKY TFs, 40 and 25 were up-regulated, while only 5 and 10 were down-regulated respectively. PIFs, a group of basic helix-loop-helix (bHLH) family, have been demonstrated as major transcription factors orchestrating dark-induced senescence [18]. A total of three PIFs were identified with significantly different expressions, including two PIF4s and one PIF3 (Table 4). The expression levels of these three PIFs were all suppressed in dark-induced senescent leaves. To investigate the expression pattern of CdPIF3/4 during dark-induced senescence, expression levels of one PIF3 and one PIF4 were further analyzed by quantitative real-time PCR (qRT-PCR) after $0,1,2,3,5$, and $7 \mathrm{~d}$ of dark treatment (Figure S2). Both CdPIF3 and CdPIF4 were highly induced after the first five days' dark treatment, but reduced after $7 \mathrm{~d}$ of dark treatment. In addition, corresponding to the numerous Hsps which were up-regulated in senescent leaves induced by darkness, a total of six heat shock transcription factors (Hsfs) with up-regulated expression were also identified (Table S5). 
Table 4. The differentially expressed phytochrome-interacting factors (PIFs) transcription factors (TFs) between CK and DT.

\begin{tabular}{cccc}
\hline GeneID & $\log _{2}(\mathrm{DT} / \mathrm{CK})$ & padj-value & Gene Description \\
\hline Cluster-109720.12769-2R & -2.8599 & $1.71 \times 10^{-16}$ & $\begin{array}{c}\text { Transcription factor PIF4 OS } \\
\text { = Arabidopsis thaliana } \mathrm{GN}= \\
\text { PIF4 PE }=1 \mathrm{SV}=1\end{array}$ \\
Cluster-107104.0-1F & -7.2349 & $1.02 \times 10^{-19}$ & $\begin{array}{c}\text { Transcription factor PIF4 OS } \\
=\text { Arabidopsis thaliana } \mathrm{GN}= \\
\text { PIF1 PE }=1 \mathrm{SV}=1\end{array}$ \\
Cluster-113186.0-2F & -5.49 & $\begin{array}{c}\text { Transcription factor PIF3 OS } \\
\text { = Oryza sativa subsp. } \\
\text { japonica GN }=\text { PIF3 PE }=1 \\
\text { SV }=1\end{array}$ \\
\hline
\end{tabular}

\subsection{Validating the DEGs by $q R T-P C R$ Analysis}

To validate the results of RNA sequencing, six DEGs including CdPIF3, CdPIF4, CdNAC092, $C d W R K Y 9, C d W R K Y 22$, and CdNAC21/22 were randomly selected for qRT-PCR. The qRT-PCR results were basically consistent with those of RNA sequencing, suggesting that the RNA sequencing results were reliable (Figure 6).
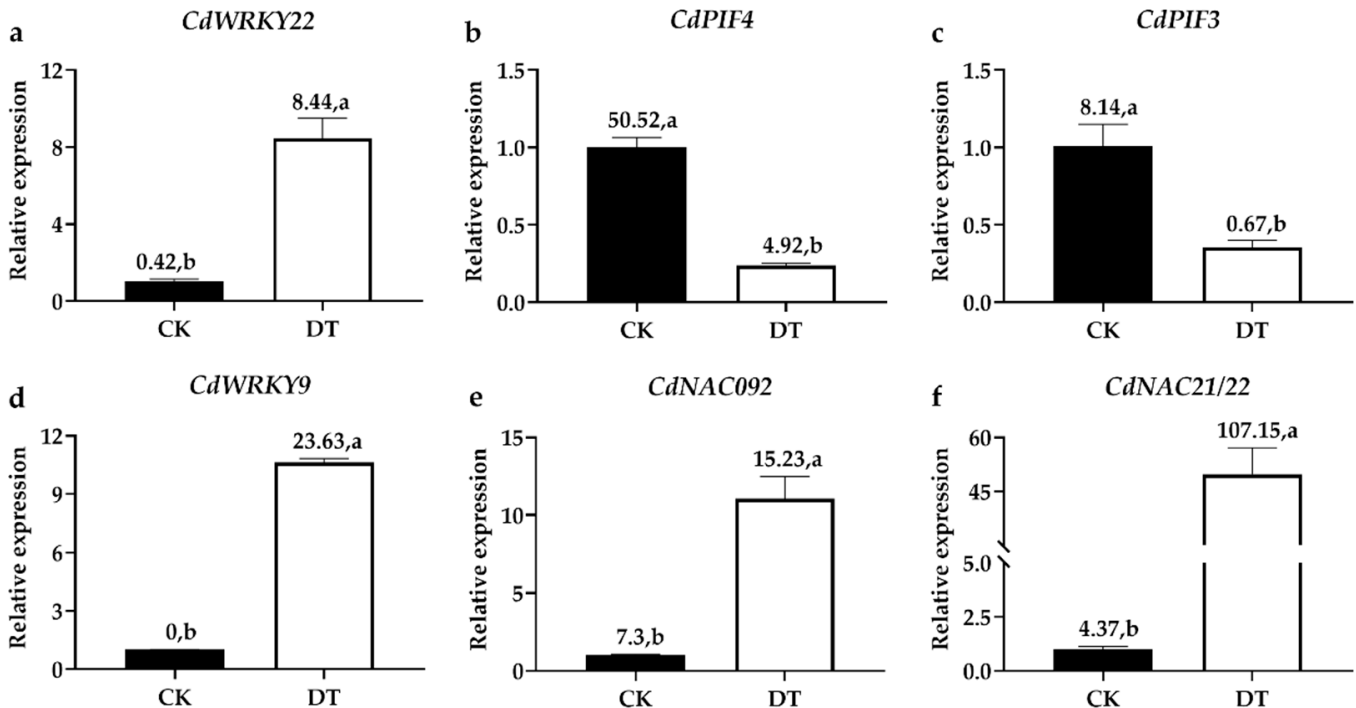

Figure 6. Real-Time RT-PCR (qRT-PCR) validation of DEGs in response to dark-induced leaf senescence. Expression analysis of (a) CdWRKY22; (b) CdPIF4; (c) CdPIF3; (d) CdWRKY9; (e) CdNAC092; (f) $C d N A C 21 / 22$ between CK and DT by qRT-PCR. The numbers above the error bars represent the fragments per kilobase of transcript per million fragments mapped (FPKM) obtained by RNA-sequence in CK and DT. Letters above error bars indicate a significant difference $(p<0.05)$ between CK and DT using Student's t-test. The error bars represent the SD value $(n=3)$.

\section{Discussion}

In this study, RNA sequencing was performed in CK and DT of bermudagrass. Our results suggested that dark responsive genes were mainly involved in plant hormone signal transduction, $\mathrm{N}$-glycan biosynthesis, and protein processing in the endoplasmic reticulum. In addition, NAC, WRKY, bHLH, and HSF TF families were also responsive to dark-induced leaf senescence in bermudagrass.

PIFs have been demonstrated as master transcription factors during dark-induced leaf senescence [18]. Among the seven PIFs in Arabidopsis, PIF3, PIF4, and PIF5 function as the central and essential transcriptional activators of dark-induced senescence $[19,20]$. Their transcriptional and 
protein levels were both induced by darkness. In this study, significantly different expressions of three PIFs including one PIF3 and two PIF4s were detected between CK and DT in bermudagrass (Table 4). However, their expression levels were all suppressed by $7 \mathrm{~d}$ of dark treatment. This was probably because PIF3, 4, and 5 are early senescence responsive genes. Their expression levels were induced at the first two days of dark treatment but reduced at day 3 post-treatment in Arabidopsis [20]. In Solanum lycopersicum, SIPIF4 also exhibited expression tendency similar to that reported for A. thaliana homologs during dark-induced senescence [34]. Further qRT-PCR results demonstrated that the expression levels of CdPIF3/PIF4 were indeed induced by short-time darkness and then declined after long-time darkness in bermudagrass, indicating that the CdPIF3/4 are early dark-induced senescence responsive genes (Figure S2).

PIFs were reported to regulate dark-induced leaf senescence by targeting hormone signaling and production. They were shown to promote dark-induced senescence by inducing both ethylene biosynthesis and signaling, and ABA signal transduction via 1-aminocyclopropane-1-carboxylate synthases (ACSs), EIN3, EEL, and ABI5 in Arabidopsis [19,35-38]. In contrast, the expression levels of CdACS, $C d E I N 3, C d E E L$, and CdABI5 were not up- or down-regulated during dark-induced senescence in bermudagrass. However, other genes involved in ethylene and ABA signaling were identified. For example, EIN2, a positive regulator of aging-induced leaf senescence [39-41], was largely induced, although EIN2 was not detected on the array of senescent leaves induced by dark treatment in Arabidopsis [42]. In addition to ethylene and ABA, other plant hormones, including JA, SA, cytokinin, auxin, gibberellin, and brassinosteroid, which have not been reported to be targeted by PIFs also participated in dark-induced leaf senescence in bermudagrass. Cytokinin can delay leaf senescence and the level of cytokinin is reduced in senescent leaves. Here, most of $C d A H K 4 s$, cytokinin receptors were induced except one $C d A H K 4$. In the cytokinin $\mathrm{AHK} \rightarrow \mathrm{AHP} \rightarrow \mathrm{ARR}$ phosphorelay pathway, A-type $A R R s$ are reported to be rapidly up-regulated by cytokinin [43]. Due to the decline of cytokinin during senescence, ARRs were observed to be down-regulated in the previous studies [6,42]. In contrast, three of four ARRs were significantly induced in this study, suggesting that they may have a special function of cytokinin signaling during dark-induced leaf senescence in bermudagrass. A similar phenomenon happened to AtARR16, which was also up-regulated during dark-induced leaf senescence in Arabidopsis [42]. JA plays a pivotal role in dark-induced leaf senescence. JAZ genes were found to be significantly induced under dark treatment, and several $J A Z$ genes, such as $J A Z 7$, were reported to negatively regulate dark-induced leaf senescence in Arabidopsis [44,45]. Consistently, all JAZ genes were induced during dark-induced leaf senescence in bermudagrass. In addition, the SA signaling pathway was also enriched in dark-induced senescence in bermudagrass, although it was not detected in Arabidopsis [6].

Many transcription factors have been identified as master senescence regulators. During dark-induced leaf senescence, the senescence key regulator AtNAC092 is activated by PIF3/4/5, EIN3, ABI5, and EEL [19]. In our study, we found CdNAC092 was significantly induced although the expression levels of CdPIF3/4 were down-regulated. Another two senescence master regulators WRKY 22 and NAP are also affected by PIFs directly or indirectly in Arabidopsis during dark-induced leaf senescence $[8,29,36]$. Here, $C d W R K Y 22$ and $C d N A C 029$ were significantly induced under dark-induced leaf senescence as well. These results suggest that the senescence master regulators might play roles during dark-induced senescence through additional PIF-independent ways.

It has been reported that $\mathrm{N}$-glycan alteration is associated with age in humans [46]. In plants, $\mathrm{N}$-glycans were reported to play an important role during fruit ripening. Two ripening-specific $\mathrm{N}$-glycoprotein modifying enzymes, $\alpha$-mannosidase ( $\alpha$-Man) and $\beta$-D-N-acetylhexosaminidase $(\beta-\mathrm{Hex})$ have been identified [47]. However, the role of N-glycan in leaf aging, especially dark-induced leaf senescence is unclear. In this study, genes involved in N-glycan biosynthesis were almost all significantly induced during dark-induced leaf senescence in bermudagrass, indicating that $\mathrm{N}$-glycan might be associated with dark-induced leaf senescence in bermudagrass. 
HSPs are molecular chaperones and have been implicated in longevity and aging in many species, such as Caenorhabditis elegans [28]. However, their association with leaf aging, especially dark-induced leaf senescence is unknown. In this study, it is interesting to note that numerous Hsps were induced during dark-induced leaf senescence, indicating that Hsps may refold the damaged proteins accumulating during dark-induced leaf senescence to maintain protein homeostasis and longevity. As we know, the expression of Hsp genes is controlled by Hsfs. As expected, several Hsfs were indeed up-regulated in the senescent leaves induced by darkness here. It was reported that overexpression of HaHsf9 resulted in a slowing of dark-induced seedling senescence [48]. The function of $\mathrm{CdHsfs}$ in dark-induced leaf senescence is yet to be revealed.

\section{Conclusions}

In this study, to better understand the mechanism of dark-induced leaf senescence in bermudagrass, the comprehensive differences in transcription levels between CK and DT in bermudagrass were investigated by RNA-sequencing. A total of 59,062 DEGs, including 52,382 up-regulated and 6680 down-regulated genes were identified as being significantly responsive to dark-induced leaf senescence. Bioinformatics analysis (Figure 7) revealed that these DEGs were mainly associated with plant hormone signal transduction (CdEIN2, CdSnRK2, CdJAZs, CdAHK4), N-glycan biosynthesis (CdALG, CdSTT,

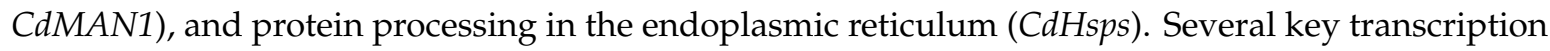
factors (CdPIF3/4, CdWRKY22, CdNAC029, and CdNAC092) were also identified during dark-induced leaf senescence. This study provides important information for the identification of genes involved in dark-induced leaf senescence in bermudagrass. Further function analyses of these candidate genes are required to reveal the molecular mechanisms underlying this complex and highly coordinated developmental process.

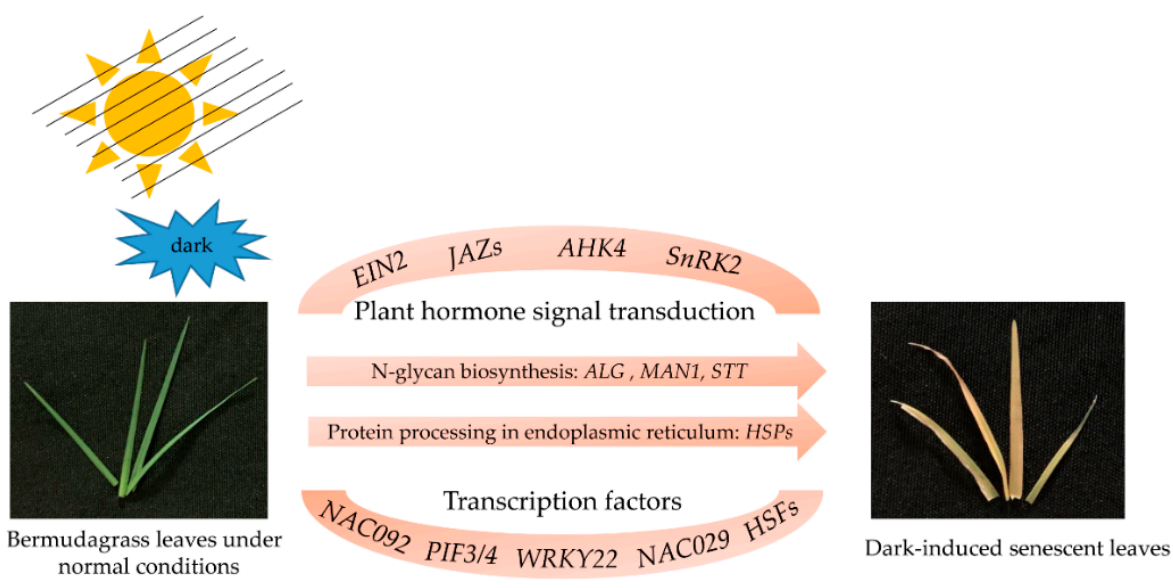

Figure 7. The mode pattern of dark/shade-induced leaf senescence in bermudagrass.

\section{Materials and Methods}

\subsection{Plant Materials and Treatments}

The bermudagrass used in this study was collected from a wild field, Zhejiang province, China. To ensure the same genetic background, stolons collected from one plant were planted in plastic pots ( $15 \mathrm{~cm}$ diameter $\times 20 \mathrm{~cm}$ tall) filled with matrix (brown coal soil:sand $=1: 1)$ to establish the seedlings. The plants were cultivated in a controlled-environment growth chamber (HP300GS-C, Ruihua Instrument, Wuhan, China), with a $16 \mathrm{~h}$ photoperiod, photosynthetically active radiation at $450 \mu \mathrm{mol} \mathrm{m} \mathrm{m}^{-2} \mathrm{~s}^{-1}$ at the canopy level, a day/night temperature of $28 / 24^{\circ} \mathrm{C}$ and $70 \%$ relative humidity. After two months of establishment, the fully developed bermudagrass plants were divided into two groups. Group I was maintained in the same chamber with the same growth conditions. Group II 
was transferred to another controlled-environment growth chamber with the same temperature and humidity but no light. One week later, green leaves were collected $4 \mathrm{~h}$ after the beginning of the light period from Group I (named as CK), and dark-induced senescent leaves were collected from Group II (named as DT). Leaf samples were collected and frozen immediately with liquid nitrogen, and stored at $-80{ }^{\circ} \mathrm{C}$ for future analysis. All the treatments were repeated for three replications.

\subsection{RNA Preparation}

The total RNA of bermudagrass leaf was extracted by Trizol reagent (Invitrogen, Carlsbad, CA, USA) according to the manufacturer's protocol. The NanoPhotometer ${ }^{\circledR}$ spectrophotometer (IMPLEN, Westlake Village, CA, USA) was applied to check the RNA purity. To guarantee the RNA purity, degradation, and contamination of RNA were monitored on $1 \%$ agarose gels. The Qubit ${ }^{\circledR}$ RNA Assay Kit was applied to measure the RNA concentration in Qubit ${ }^{\circledR} 2.0$ Fluorometer (Life Technologies, Carlsbad, CA, USA). The RNA Nano 6000 Assay Kit of the Agilent Bioanalyzer 2100 system (Agilent Technologies, Santa Clara, CA, USA) was applied to detect the RNA integrity.

\subsection{Library Preparation for Transcription Sequencing}

A total of $1.5 \mu \mathrm{g}$ RNA per sample was used for library preparation. The sequencing libraries were generated by NEBNext ${ }^{\circledR}$ Ultra ${ }^{\mathrm{TM}}$ RNA Library Prep Kit for Illumina ${ }^{\circledR}$ (NEB, Ipswich, MA, USA) according to the manufacturer's recommendations. Simultaneously, the index codes were added for attributing sequences to each sample. In brief, after mRNA purification from total RNA with poly-T oligo-attached magnetic beads, the fragmentation was carried out under elevated temperature by divalent cations in NEBNext First Strand Synthesis Reaction Buffer. Then random hexamer primer and M-MuLV Reverse Transcriptase (RNase $\left.\mathrm{H}^{-}\right)$was applied to the synthesized first-strand cDNA, and DNA polymerase I and RNase $\mathrm{H}$ were used to obtain the second strand DNA. The remaining overhangs were converted to blunt ends by exonuclease/polymerase. The NEBNext Adaptor with a hairpin loop structure was ligated to adenylated 3' ends of DNA fragments to prepare for hybridization reaction. During this process, the fragments were purified by AMPure XP system (Beckman Coulter, Beverly, CA, USA) to select the cDNA fragments in length of 250-300 bp. Before PCR, $3 \mu L$ USER Enzyme (NEB, Ipswich, MA, USA) was applied with size-selected, adaptor-ligated cDNA for 15 min at $37^{\circ} \mathrm{C}$ followed by $5 \mathrm{~min}$ at $95^{\circ} \mathrm{C}$. To perform PCR, Universal PCR primers, Index (X) Primer, and Phusion High-Fidelity DNA polymerase were used. The PCR products were purified with AMPure XP system and the library quality was estimated with Agilent Bioanalyzer 2100 system.

The clustering of the index-coded samples was conducted by the cBot Cluster Generation System with the application of TruSeq PE Cluster Kit v3-cBot-HS (Illumina, San Diego, CA, USA). After that, the samples were sequenced on an Illumina Hiseq platform.

\subsection{Data Analysis}

\subsubsection{Quality Control}

To obtain the clean data, the reads containing adapter and ploy- $\mathrm{N}$ as well as low-quality reads were removed from the raw data. Simultaneously, Q20, Q30, GC-content and sequence duplication levels of the clean data were calculated. The high-quality clean data was used for downstream analyses.

\subsubsection{Transcriptome Assembly}

Transcriptome assembly was performed with Trinity [49], min_kmer_cov was set to 2 by default and other parameters were set to default.

\subsubsection{Gene Functional Annotation}

Gene functions were annotated based on the databases of Nr (NCBI nonredundant protein sequences, ftp://ftp.ncbi.nih.gov/blast/db), Nt (NCBI nonredundant nucleotide sequences, ftp://ftp.ncbi. 
nih.gov/blast/db), Pfam (Protein family, http://pfam.sanger.ac.uk/), KOG/COG (Clusters of Orthologous Groups of proteins, http://www.ncbi.nlm.nih.gov/COG/), Swiss-Prot (A manually annotated and reviewed protein sequence database, http://www.ebi.ac.uk/uniprot/), KO (KEGG Ortholog database, http://www.genome.jp/kegg/) and GO (Gene Ontology, http://www.geneontology.org/).

\subsubsection{Differential Expression Analysis}

Differential expression analysis was performed by the DESeq R package (1.10.1). To control the false discovery rate, the Benjamini and Hochberg's approach was applied to adjust the resulting $p$ values. Genes with an adjusted $p$-value $<0.05$ were assigned as differentially expressed genes (DEGs).

\subsubsection{GO Enrichment Analysis}

Gene Ontology (GO) enrichment analysis of the DEGs was conducted by the GOseq R packages which were based on Wallenius noncentral hypergeometric distribution [50].

\subsubsection{KEGG Pathway Enrichment Analysis}

For KEGG pathway enrichment analysis, KOBAS software was applied to test the statistical enrichment of DEGs in KEGG pathways [51].

\subsection{Validation of RNA-Sequence Data by Quantitative Real-Time PCR ( $q R T-P C R$ )}

The expression levels of DEGs were performed by qRT-PCR following the previously described method [52]. In brief, cDNA synthesis was performed using a PrimeScript RT reagent kit (Takara, Dalian, China). The qRT-PCR was performed using $20 \mu \mathrm{L}$ volumes of SYBR (Takara, Dalian, China) and the ABI STEPONE Real-Time PCR system. The thermal cycle parameters used were as follows: $30 \mathrm{~s}$ at $95^{\circ} \mathrm{C}, 40$ cycles of $10 \mathrm{~s}$ at $95^{\circ} \mathrm{C}$, and $30 \mathrm{~s}$ at $60^{\circ} \mathrm{C}$. To confirm that only one PCR product was amplified and detected, a melting curve analysis of amplification products was performed at the end of each PCR reaction. CdACTIN2 was selected as a reference gene for normalization. All gene-specific primers used for qRT-PCR analysis are listed in Table S6. Three independent biological replicates were used for qRT-PCR analysis.

\subsection{Measurements of Chlorophyll Content and Fv/Fm}

The leaves were cut into small pieces and incubated in $5 \mathrm{~mL}$ dimethylsulfoxide for $12 \mathrm{~h}$ under dark conditions for chlorophyll extraction. Chlorophyll was detected by measuring absorption (OD) at 649 and $665 \mathrm{~nm}$ with a spectrophotometer (UV-2600, UNICO, Dayton, NJ, USA), and chlorophyll content $\left(\mathrm{mg} \cdot \mathrm{g}^{-1} \mathrm{FW}\right)$ was calculated as $(18.08 \times$ OD649 $+6.63 \times$ OD665 $) \times 0.005 / \mathrm{W}$.

Before measuring Fv/Fm, leaves were kept in the dark for 30 min to close all PSII reaction centers. Then Fv/Fm was measured by a pulse-amplitude modulation (PAM) portable chlorophyll fluorometer (PAM- 2500, WALZ, Effeltrich, Germany) according to the manufacturer's protocol.

Supplementary Materials: The following are available online at http://www.mdpi.com/2223-7747/8/12/614/s1. Table S1. Gene Ontology (GO) classification of DEGs between CK and DT. Table S2. DEGs involved in signal transduction of gibberellin, and brassinosteroid. Table S3. The differentially expressed NAC TFs between CK and DT. Table S4. The differentially expressed WRKY TFs between CK and DT. Table S5. The differentially expressed HSF TFs between CK and DT. Table S6. All primers used in this study. Figure S1. Kyoto encyclopedia of genes and genomes (KEGG) analysis of DEGs between CK and DT. (a) KEGG analysis of up-regulated DEGs; (b) KEGG analysis of down-regulated DEGs. Figure S2. qRT-PCR analysis of CdPIF3 and CdPIF4 during dark-induced leaf senescence. Relative expression of (a) CdPIF3; (b) CdPIF4 in the first and second leaves from bermudagrass after $1,1,2,3,5$ and $7 \mathrm{~d}$ of dark treatments. Letters above error bars indicate significant difference $(p<0.05)$ using Tukey's HSD test. The error bars represent the SD value $(n=3)$.

Author Contributions: L.C. (Liang Chen) and J.F. designed the experiment; L.C. (Liwen Cao) analyzed the data; L.C. (Liwen Cao) and J.F. wrote the manuscript; Y.L. and H.S. revised the manuscript. 
Funding: This research was financially supported by the National Natural Science Foundation of China (Grant No. 31702165, 31672482), General Financial Grant from the China Postdoctoral Science Foundation (Grant No. 2017M621838).

Conflicts of Interest: The authors have no conflicts of interest to declare.

\section{References}

1. Peterson, K.W.; Fry, J.D.; Bremer, D.J. Growth Responses of Zoysia spp. under Tree Shade in the Midwestern United States. HortScience 2014, 49, 1444-1448. [CrossRef]

2. Tegg, R.S.; Lane, P.A. A comparison of the performance and growth of a range of turfgrass species under shade. Aust. J. Exp. Agric. 2004, 44, 353-358. [CrossRef]

3. Chin, S.-W. Comparative Performance of Three Tropical Turfgrasses Digitaria longiflora, Axonopus compressus and St. Augustinegrass under Simulated Shade Conditions. Weed Turfgrass Sci. 2017, 6, 55-60. [CrossRef]

4. Lim, P.O.; Woo, H.R.; Gil Nam, H. Molecular genetics of leaf senescence in Arabidopsis. Trends Plant Sci. 2003, 8, 272-278. [CrossRef]

5. Brouwer, B.; Ziolkowska, A.; Bagard, M.; Keech, O.; Gardeström, P. The impact of light intensity on shade-induced leaf senescence. Plant Cell Environ. 2012, 35, 1084-1098. [CrossRef] [PubMed]

6. Buchanan-Wollaston, V.; Page, T.; Harrison, E.; Breeze, E.; Lim, P.O.; Gil Nam, H.; Lin, J.-F.; Wu, S.-H.; Swidzinski, J.; Ishizaki, K.; et al. Comparative transcriptome analysis reveals significant differences in gene expression and signalling pathways between developmental and dark/starvation-induced senescence in Arabidopsis. Plant J. 2005, 42, 567-585. [CrossRef]

7. Lee, R.; Wang, C.; Huang, L.; Chen, S.G. Leaf senescence in rice plants: Cloning and characterization of senescence up-regulated genes. J. Exp. Bot. 2001, 52, 1117-1121. [CrossRef]

8. Yang, J.; Worley, E.; Udvardi, M. A NAP-AAO3 regulatory module promotes chlorophyll degradation via ABA biosynthesis in Arabidopsis leaves. Plant Cell 2014, 26, 4862-4874. [CrossRef]

9. Guo, Y.; Gan, S. AtNAP, a NAC family transcription factor, has an important role in leaf senescence. Plant J. 2006, 46, 601-612. [CrossRef]

10. Kim, H.; Kim, H.J.; Vu, Q.T.; Jung, S.; McClung, C.R.; Hong, S.; Gil Nam, H. Circadian control of ORE1 by PRR9 positively regulates leaf senescence in Arabidopsis. Proc. Natl. Acad. Sci. USA 2018, 115, 8448-8453. [CrossRef]

11. Zhuo, M.; Sakuraba, Y.; Yanagisawa, S. A Jasmonate-activated MYC2-Dof2.1-MYC2 Transcriptional Loop Promotes Leaf Senescence in Arabidopsis. Plant Cell 2019. [CrossRef] [PubMed]

12. Liang, C.; Wang, Y.; Zhu, Y.; Tang, J.; Hu, B.; Liu, L.; Ou, S.; Wu, H.; Sun, X.; Chu, J.; et al. OsNAP connects abscisic acid and leaf senescence by fine-tuning abscisic acid biosynthesis and directly targeting senescence-associated genes in rice. Proc. Natl. Acad. Sci. USA 2014, 111, 10013-10018. [CrossRef] [PubMed]

13. Kusaba, M.; Ito, H.; Morita, R.; Iida, S.; Sato, Y.; Fujimoto, M.; Tanaka, A. Rice Non-Yellow Coloring1 is involved in light-harvesting complex II and grana degradation during leaf senescence. Plant Cell 2007, 19, 1362-1375. [CrossRef] [PubMed]

14. Morita, R.; Sato, Y.; Masuda, Y.; Nishimura, M.; Kusaba, M. Defect in non-yellow coloring 3, an $\alpha / \beta$ hydrolase-fold family protein, causes a stay-green phenotype during leaf senescence in rice. Plant J. 2009, 59, 940-952. [CrossRef] [PubMed]

15. Sakuraba, Y.; Han, S.H.; Yang, H.J.; Piao, W.; Paek, N.C. Mutation of Rice Early Flowering3. 1 (OsELF3. 1) delays leaf senescence in rice. Plant Mol. Biol. 2016, 92, 223-234. [CrossRef] [PubMed]

16. Breeze, E.; Harrison, E.; McHattie, S.; Hughes, L.; Hickman, R.; Hill, C.; Kiddle, S.; Kim, Y.-S.; Penfold, C.A.; Jenkins, D.; et al. High-resolution temporal profiling of transcripts during Arabidopsis leaf senescence reveals a distinct chronology of processes and regulation. Plant Cell 2011, 23, 873-894. [CrossRef]

17. Kanazawa, S.; Sano, S.; Koshiba, T.; Ushimaru, T. Changes in antioxidative enzymes in cucumber cotyledons during natural senescence: Comparison with those during dark-induced senescence. Physiol. Plant. 2000, 109, 211-216. [CrossRef]

18. Liebsch, D.; Keech, O. DarK-induced leaf senescence: New insights into a complex light-dependent regulatory pathway. New Phytol. 2016, 212, 563-570. [CrossRef] 
19. Sakuraba, Y.; Jeong, J.; Kang, M.-Y.; Kim, J.; Paek, N.-C.; Choi, G. Phytochrome-interacting transcription factors PIF4 and PIF5 induce leaf senescence in Arabidopsis. Nat. Commun. 2014, 5, 4636. [CrossRef]

20. Song, Y.; Yang, C.; Gao, S.; Zhang, W.; Li, L.; Kuai, B. Age-triggered and dark-induced leaf senescence require the bHLH transcription factors PIF3, 4, and 5. Mol. Plant 2014, 7, 1776-1787. [CrossRef]

21. Qiu, K.; Li, Z.; Yang, Z.; Chen, J.; Wu, S.; Zhu, X.; Gao, S.; Gao, J.; Ren, G.; Kuai, B.; et al. EIN3 and ORE1 Accelerate Degreening during Ethylene-Mediated Leaf Senescence by Directly Activating Chlorophyll Catabolic Genes in Arabidopsis. PLoS Genet. 2015, 11, e1005399. [CrossRef] [PubMed]

22. Dietrich, K.; Weltmeier, F.; Ehlert, A.; Weiste, C.; Stahl, M.; Harter, K.; Dröge-Laser, W. Heterodimers of the Arabidopsis Transcription Factors bZIP1 and bZIP53 Reprogram Amino Acid Metabolism during Low Energy Stress. Plant Cell 2011, 23, 381-395. [CrossRef] [PubMed]

23. Mair, A.; Pedrotti, L.; Wurzinger, B.; Anrather, R.; Simeunovic, A.; Weiste, C.; Valerio, C.; Dietrich, K.; Kirchler, T.; Nagele, T.; et al. Decision letter: SnRK1-triggered switch of bZIP63 dimerization mediates the low-energy response in plants. Decis. Lett. 2015, 4, 05828.

24. Huang, X.; Shi, H.; Hu, Z.; Liu, A.; Amombo, E.; Chen, L.; Fu, J. ABA Is Involved in Regulation of Cold Stress Response in Bermudagrass. Front. Plant Sci. 2017, 8, 1613. [CrossRef] [PubMed]

25. Hieno, A.; Naznin, H.A.; Inaba-Hasegawa, K.; Yokogawa, T.; Hayami, N.; Nomoto, M.; Tada, Y.; Yokogawa, T.; Higuchi-Takeuchi, M.; Hanada, K.; et al. Transcriptome Analysis and Identification of a Transcriptional Regulatory Network in the Response to $\mathrm{H}_{2} \mathrm{O}_{2}$. Plant Physiol. 2019, 180, 1629-1646. [CrossRef] [PubMed]

26. Kumar, M.; Kim, I.; Kim, Y.-K.; Heo, J.B.; Suh, M.C.; Kim, H.U. Strigolactone Signaling Genes Showing Differential Expression Patterns in Arabidopsis max Mutants. Plants 2019, 8, 352. [CrossRef] [PubMed]

27. Kumar, M.; Gho, Y.-S.; Jung, K.-H.; Kim, S.-R. Genome-Wide Identification and Analysis of Genes, Conserved between japonica and indica Rice Cultivars, that Respond to Low-Temperature Stress at the Vegetative Growth Stage. Front. Plant Sci. 2017, 8, 1120. [CrossRef]

28. Murshid, A.; Eguchi, T.; Calderwood, S.K. Stress proteins in aging and life span. Int. J. Hyperth. 2013, 29, 442-447. [CrossRef]

29. Zhou, X.; Jiang, Z.; Yu, D. WRKY22 Transcription Factor Mediates Dark-Induced Leaf Senescence in Arabidopsis. Mol. Cells 2011, 31, 303-313. [CrossRef]

30. Guo, P.; Li, Z.; Huang, P.; Li, B.; Fang, S.; Chu, J.; Guo, H. A Tripartite Amplification Loop Involving the Transcription Factor WRKY75, Salicylic Acid, and Reactive Oxygen Species Accelerates Leaf Senescence. Plant Cell 2017, 29, 2854-2870. [CrossRef]

31. Miao, Y.; Laun, T.; Zimmermann, P.; Zentgraf, U. Targets of the WRKY53 transcription factor and its role during leaf senescence in Arabidopsis. Plant Mol. Boil. 2004, 55, 853-867. [CrossRef]

32. Balazadeh, S.; Kwasniewski, M.; Caldana, C.; Mehrnia, M.; Zanor, M.I.; Xue, G.-P.; Mueller-Roeber, B. ORS1, an $\mathrm{H}_{2} \mathrm{O}_{2}$-Responsive NAC Transcription Factor, Controls Senescence in Arabidopsis thaliana. Mol. Plant 2011, 4, 346-360. [CrossRef] [PubMed]

33. Balazadeh, S.; Siddiqui, H.; Allu, A.D.; Matallana-Ramirez, L.P.; Caldana, C.; Mehrnia, M.; Köhler, B.; Mueller-Roeber, B.; Zanor, M.-I. A gene regulatory network controlled by the NAC transcription factor ANAC092/AtNAC2/ORE1 during salt-promoted senescence. Plant J. 2010, 62, 250-264. [CrossRef] [PubMed]

34. Rosado, D.; Gramegna, G.; Cruz, A.; Lira, B.S.; Freschi, L.; De Setta, N.; Rossi, M. Phytochrome Interacting Factors (PIFs) in Solanum lycopersicum: Diversity, Evolutionary History and Expression Profiling during Different Developmental Processes. PLoS ONE 2016, 11, e0165929. [CrossRef] [PubMed]

35. Cho, Y.-H.; Yoo, S.-D. Novel connections and gaps in ethylene signaling from the ER membrane to the nucleus. Front. Plant Sci. 2015, 5, 733. [CrossRef]

36. Zhang, Y.; Liu, Z.; Chen, Y.; He, J.-X.; Bi, Y. Phytochrome-Interacting Factor 5 (PIF5) positively regulates dark-induced senescence and chlorophyll degradation in Arabidopsis. Plant Sci. 2015, 237, 57-68. [CrossRef]

37. Li, Z.; Peng, J.; Wen, X.; Guo, H. Ethylene-Insensitive3 Is a Senescence-Associated Gene That Accelerates Age-Dependent Leaf Senescence by Directly Repressing miR164 Transcription in Arabidopsis. Plant Cell 2013, 25, 3311-3328. [CrossRef]

38. Khanna, R.; Shen, Y.; Marion, C.M.; Tsuchisaka, A.; Theologis, A.; Schäfer, E.; Quail, P.H. The Basic Helix-Loop-Helix Transcription Factor PIF5 Acts on Ethylene Biosynthesis and Phytochrome Signaling by Distinct Mechanisms. Plant Cell 2007, 19, 3915-3929. [CrossRef]

39. Niu, Y.-H.; Guo, F.-Q. Nitric Oxide Regulates Dark-Induced Leaf Senescence Through EIN2 in ArabidopsisF. J. Integr. Plant Boil. 2012, 54, 516-525. [CrossRef] 
40. Kim, H.J.; Hong, S.H.; Kim, Y.W.; Lee, I.H.; Jun, J.H.; Phee, B.-K.; Rupak, T.; Jeong, H.; Lee, Y.; Hong, B.S.; et al. Gene regulatory cascade of senescence-associated NAC transcription factors activated by Ethylene-Insensitive2-mediated leaf senescence signalling in Arabidopsis. J. Exp. Bot. 2014, 65, 4023-4036. [CrossRef]

41. Oh, S.A.; Park, J.; Lee, G.I.; Paek, K.H.; Park, S.K.; Gil Nam, H.; Miklashevichs, E.; Czaja, I.; Cordeiro, A.; Prinsen, E.; et al. Identification of three genetic loci controlling leaf senescence in Arabidopsis thaliana. Plant J. 1997, 12, 527-535. [CrossRef] [PubMed]

42. Van Der Graaff, E.; Schwacke, R.; Schneider, A.; DeSimone, M.; Flügge, U.-I.; Kunze, R. Transcription Analysis of Arabidopsis Membrane Transporters and Hormone Pathways during Developmental and Induced Leaf Senescence1. Plant Physiol. 2006, 141, 776-792. [CrossRef] [PubMed]

43. Kiba, T.; Naitou, T.; Koizumi, N.; Yamashino, T.; Sakakibara, H.; Mizuno, T. Combinatorial Microarray Analysis Revealing Arabidopsis Genes Implicated in Cytokinin Responses through the His $\rightarrow$ Asp Phosphorelay Circuitry. Plant Cell Physiol. 2005, 46, 339-355. [CrossRef] [PubMed]

44. Yu, J.; Zhang, Y.; Di, C.; Zhang, Q.; Zhang, K.; Wang, C.; You, Q.; Yan, H.; Dai, S.Y.; Yuan, J.S.; et al. JAZ7 negatively regulates dark-induced leaf senescence in Arabidopsis. J. Exp. Bot. 2015, 67, 751-762. [CrossRef] [PubMed]

45. Lin, J.F.; Wu, S.H. Molecular events in senescing Arabidopsis leaves. Plant J. 2004, 39, 612-628. [CrossRef]

46. Ding, N.; Nie, H.; Sun, X.; Sun, W.; Qu, Y.; Liu, X.; Yao, Y.; Liang, X.; Chen, C.C.; Li, Y. Human serum N-glycan profiles are age and sex dependent. Age Ageing 2011, 40, 568-575. [CrossRef]

47. Meli, V.S.; Ghosh, S.; Prabha, T.N.; Chakraborty, N.; Chakraborty, S.; Datta, A. Enhancement of fruit shelf life by suppressing N-glycan processing enzymes. Proc. Natl. Acad. Sci. USA 2010, 107, 2413-2418. [CrossRef]

48. Almoguera, C.; Personat, J.-M.; Prieto-Dapena, P.; Jordano, J. Heat shock transcription factors involved in seed desiccation tolerance and longevity retard vegetative senescence in transgenic tobacco. Planta 2015, 242, 461-475. [CrossRef]

49. Grabherr, M.G.; Haas, B.J.; Yassour, M.; Levin, J.Z.; Thompson, D.A.; Amit, I.; Adiconis, X.; Fan, L.; Raychowdhury, R.; Zeng, Q.; et al. Full-length transcriptome assembly from RNA-Seq data without a reference genome. Nat. Biotechnol. 2011, 29, 644-652. [CrossRef]

50. Young, M.D.; Wakefield, M.J.; Smyth, G.K.; Oshlack, A. Gene ontology analysis for RNA-seq: Accounting for selection bias. Genome Boil. 2010, 11, R14. [CrossRef]

51. Mao, X.; Cai, T.; Olyarchuk, J.G.; Wei, L. Automated genome annotation and pathway identification using the KEGG Orthology (KO) as a controlled vocabulary. Bioinformatics 2005, 21, 3787-3793. [CrossRef] [PubMed]

52. Cao, L.; Yu, N.; Li, J.; Qi, Z.; Wang, D.; Chen, L. Heritability and Reversibility of DNA Methylation Induced by in vitro Grafting between Brassica juncea and B. oleracea. Sci. Rep. 2016, 6, 27233. [CrossRef] [PubMed] 